\title{
(Mal) Adaptações Metabólicas ao Treinamento Contínuo - Concepções não Consensuais de Terminologia e Diagnóstico
}

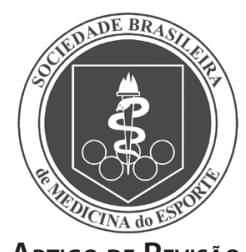

\author{
Metabolic (Mal) Adaptations to Training Continuum
}

-Misconceptions of Terminology and Diagnosis

\begin{abstract}
Franz Homero Paganini Burini ${ }^{1}, 2$ Erick Prado de Oliveira1,3

Roberto Carlos Burini $1^{1,4}$

1. Centro de Metabolismo em Exercício e Nutrição (CeMENutri) - Departamento de Saúde Pública Faculdade de Medicina de Botucatu (UNESP) - Botucatu, São Paulo/SP. 2. Pós-graduação no Pronut (USP-SP). 3. Pós-graduação em Patologia - Departamento de Patologia Faculdade de Medicina de Botucatu (UNESP) - Botucatu, São Paulo/SP. 4. Professor Titular do Departamento de Saúde Pública da Faculdade de Medicina de Botucatu (UNESP) e responsável pelo CeMENutri.
\end{abstract}

\section{Endereço para correspondência:} Prof. Dr Roberto Carlos Burini CeMENutri - Centro de Metabolismo em Exercício e Nutrição Faculdade de Medicina, Departamento de Saúde Pública (FMB-UNESP)

Distrito de Rubião $\mathrm{Jr}, \mathrm{s} / \mathrm{n}^{\circ}$; CEP: 18.618-970; Botucatu/SP E-mail: burini@fmb.unesp.br

\begin{abstract}
RESUMO
Altos desempenhos esportivos demandam treinamentos pesados necessários ao estímulo adaptativo específico a cada esporte. A elevada carga de treino é geralmente acompanhada de discreta fadiga e reduções agudas no desempenho, mas caso acompanhada de períodos apropriados de recuperação, resulta em supercompensação metabólica ao treinamento, refletida como aumento na capacidade aeróbica e/ou força muscular. Visto como contínuo, os processos de intensificação do treinamento e o estresse relacionado à supercompensação, o aumento da sobrecarga ou do estresse poderá, em algum momento, acarretar a quebra da homeostase e a queda temporária da função (supra-alcance - OR ou supra-alcance funcional - FOR). Quando a sobrecarga excessiva de treinamento é combinada com recuperação inadequada há instalação do estado de supratreinamento (OT) ou supra-alcance não funcional (NFOR). O OT excede o OR, cujo pico é também o limiar do OT resultando em desadaptações fisiológicas e queda crônica do desempenho físico. A forma crônica de desadaptação fisiológica ao treinamento físico é chamada de síndrome do supertreinamento (OTS). A própria expressão da síndrome denota a etiologia multifatorial do estado e reconhece que o exercício não é necessariamente seu único fator causal. O diagnóstico de OTS é baseado na recuperação ou não do desempenho. Não há biomarcador objetivo para OTS. A distinção entre OTS e NFOR (supratreinamento extremo) é dependente de desfecho clínico e exclusão diagnóstica de doenças orgânicas, mais comuns na OTS. Também a diferença entre OR e OT é sutil e nenhum de seus marcadores bioquímicos pode ser universalizado. Não há evidências confirmatórias que OR evolui para OT ou que os sintomas de OT são piores dos que os de OR. Apenas pela fadiga aguda e queda de rendimento experimentada em sessões isoladas de treinamento, não é possível diferenciar presentemente os estados de OR e OT. Isto é devido, parcialmente, à variabilidade das respostas individuais ao treinamento e à falta de ambos instrumentos diagnósticos e estudos bem controlados.
\end{abstract}

Palavras-chave: estresse do treinamento, desadaptação fisiológica, supra-alcance, supratreinamento.

\section{ABSTRACT}

Sports top-level performance requires heavy training loads that provide a stimulus to sport-specific adaptation. Competitive training involving high workload is generally accompanied by minor fatigue and acute performance reduction, but when followed by appropriate recovery periods results in training-metabolic supercompensation reflected as increase in aerobic capacity and muscular strength. When the intensified training process leading to supercompensation and related-stress is seen as a continuum, the increased stress or overload might result in disruption of homeostasis and temporary decrease in function (overreaching - OR or functional overreaching - FOR). When excessive overload is combined with inadequate recovery, a state of overtraining (OT) or non-functional overreaching (NFOR) is installed. OT exceeds OR and the OR peak is also the OT threshold, resulting in stark physiological maladaptations and chronically reduced exercise performance. The chronic form of physiological maladaptation to training is called overtraining syndrome (OTS). The expression of the syndrome emphasizes the multifactorial etiology of the state and acknowledges that exercise (training) is not necessarily the sole causative factor. There is no objective biomarker for OTS besides the diagnosis based on performance recovery. Other distinctions between NFOR (extreme OT) and OTS depend on clinical outcome and exclusion diagnosis of organic diseases more common in OTS. Additionally, the difference between OR and OT is subtle and none of their biochemical markers should be considered universal. There is no evidence to confirm that OR will develop into OT or that OT symptoms are worse than those of OR. It is presently not possible to differentiate OR and OT states from the acute fatigue and decreased performance experienced from isolated training sessions. This situation is partially due to the variability of individual responses to training and to a lack of both diagnostic tools and well controlled studies.

Keywords: training stress, physiological maladaptation, overreaching, overtraining. 


\section{Objetivos e desfechos do treinamento}

O objetivo dos programas de treinamento é estimular a adaptação específica ao esporte que resultará em melhora ou manutenção do desempenho atlético. Treinamento pode ser definido como processo de sobrecarga que altera a homeostase resultando em fadiga aguda, mas no geral, leva à melhoria do desempenho ${ }^{(1)}$. Sobrecarga refere-se ao aumento progressivo e sistemático do treinamento, planejado como estímulo necessário às melhorias de força, potência e resistência(2).

O treinamento competitivo, envolvendo alta sobrecarga, é geralmente acompanhado de ligeira fadiga e reduções agudas no desempenho, mas quando seguido por períodos apropriados de recuperação resulta em supercompensação metabólica refletida como aumento da capacidade aeróbica e força muscular ${ }^{(3)}$.

A melhor estratégia conhecida para aumentar a massa muscular esquelética (hipertrofia) é pelo emprego do treinamento resistido contra cargas mecânicas. A síntese proteica muscular, induzida pelo treinamento, ocorre após sobrecarga e dano/reparo tecidual(4).

Desempenho de alto nível requer, em esportes de resistência, vários anos de treinamentos pesados. O maior objetivo deste treinamento de resistência é atingir as adaptações metabólicas mais elevadas, toleradas pelo atleta ${ }^{(2)}$. Corredores, aerobicamente bem treinados, mantém não apenas ritmo prolongado, mas também velocidade moderada de recuperação para remoção continuada do lactato sanguíneo(5).

A figura 1 mostra a sequência clássica das respostas aos períodos de treinamento mesocíclicos experimentados na temporada de competição. Neste modelo os atletas vão das fases do subtreinamento (visto tanto entre temporadas como no repouso ativo) ao supratreinamento (OT) passando pelo supra-alcance $(\mathrm{OR})^{(6)}$.

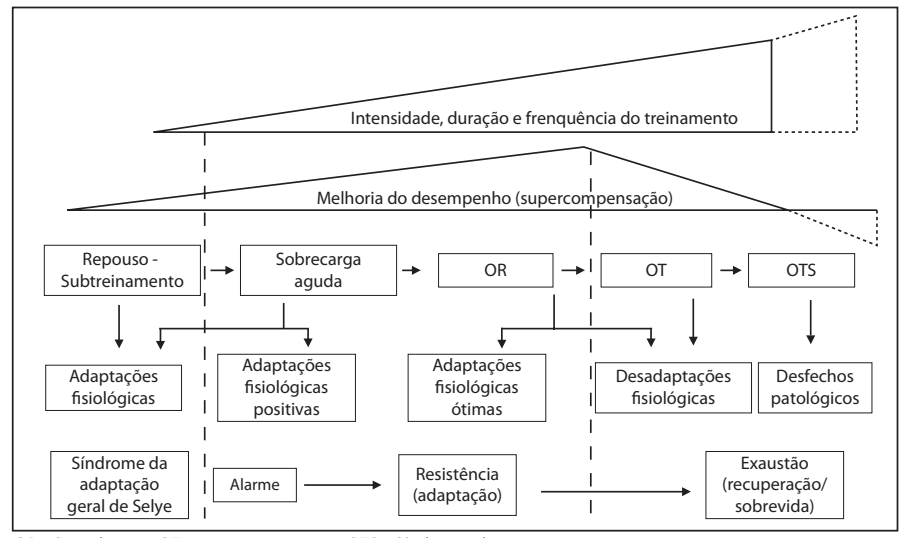

$\mathrm{OR}=$ Supralcance; $\mathrm{OT}=$ supratreinamento; OTS= Síndrome do supratreinamento

Figura 1. Estados do treinamento contínuo dentro da temporada de competições

\section{ADAPTAÇÕES FISIOLÓGICAS AO TREINAMENTO CONTÍNUO}

A figura 1 mostra que o processo de intensificação do treinamento, levando à supercompensação, pode resultar em adaptações fisiológicas ao estresse causado pelo rompimento da homeostase metabólica.

Ao estímulo do exercício, impulsos dos centros motores cerebrais, assim como dos músculos em atividade, desencadeiam a atividade simpática adrenal, sobrecarga-dependente, com a liberação de hormônios hipofisários. A maior atividade simpática é importante para a adaptação cardiovascular, termorregulação e oferta de substratos energéticos, no exercício. O débito cardíaco é aumentado enquanto a volemia e o fluxo sanguíneo são redistribuídos favorecendo os músculos ativos e a pele ${ }^{(7)}$.

A ativação central do sistema nervoso simpático (SNS), conjuntamente ao sistema motor, tem a atividade modulada de acordo com a magnitude do desfecho motor (feed-forward). Adicionalmente, o SNS responde aos sinais aferentes do músculo ativados em resposta às alterações químicas locais(8).
Concomitantemente ao estresse do treinamento, o sistema endócrino é acionado para responder à situação de estresse ${ }^{(1)}$. Neste momento, dois eixos hormonais são ativados, o simpaticomedular (adrenal) e o hipotalâmico-hipofisário-adrenal. A figura 2 mostra estes dois eixos cujos efeitos são complementares no preparo do atleta para a ação e exercício. Seus hormônios primários (epinefrina, norepinefrina e cortisol) servem para mobilizar e redistribuir os combustíveis metabólicos em diferentes velocidades, manter a glicemia e aumentar a responsividade do sistema cardiovascular ${ }^{(1,9)}$.

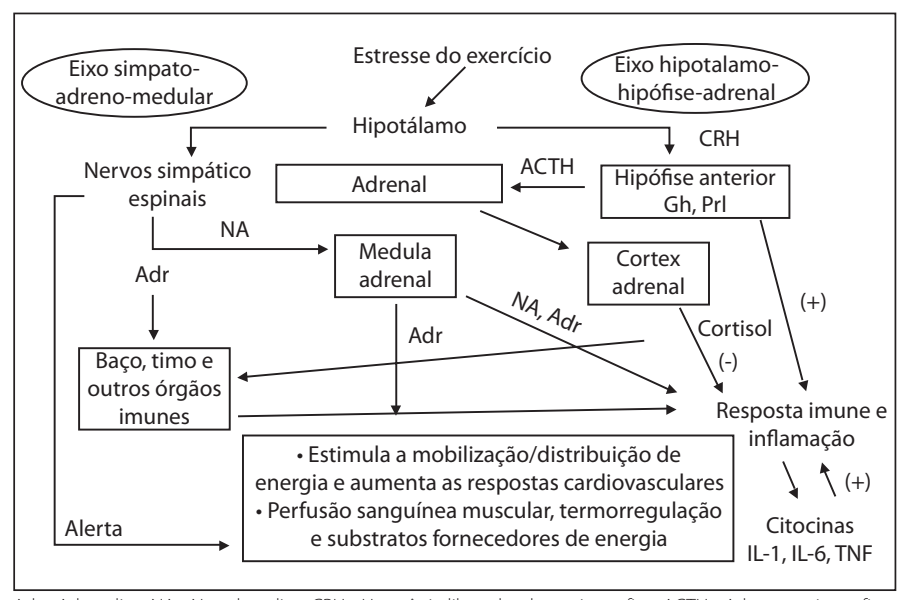

Adr= Adrenalina; $\mathrm{NA}=$ Noradrenalina; $\mathrm{CRH}=$ Hormônio liberador de corticotrofina; $\mathrm{ACTH}=$ Adrenocorticotrofina; Gh= Hormônio de crescimento; Prl = Prolactina

Figura 2. Eixos hormonais predominantes e ações envolvidas na resposta ao exercício

O SNS aumenta o suprimento de energia, promovendo glicogenólise e a lipólise, no fígado e tecido adiposo, mantém a hiperventilação pela dilatação das vias aéreas superiores e aumenta a dissipação do calor gerado pelos músculos em atividade. OSNS modula ainda várias funções da célula muscular esquelética tais como o fluxo iônico transmembrana, a liberação de acetilcolina do nervo na placa motora, liberação e recaptação de cálcio do retículo sarcoplasmático e metabolismo da proteína (inibição da proteólise) e da glicose $e^{(8)}$.

A contração muscular por intermédio das proteínas cálcio-dependentes e quinase ativada pela hidrólise do ATP (adenosina monofosfato quinase - AMPK) propicia a biogênese mitocondrial, a miogênese fibrilar tipo I, a vasodilatação endotélio-dependente, a translocação do Glut-4 e a beta oxidação dos ácidos graxos ${ }^{(10)}$. Como resultados, o treinamento de resistência (à fadiga) leva a maior capacidade aeróbica, maior oxidação de substratos e capacidade antioxidante ${ }^{(11)}$.

No treinamento resistido, a hipertrofia muscular ocorre mediante a síntese proteica miofibrilar responsiva ao estímulo da sobrecarga muscular acompanhada do dano e reparo tecidual. Logo após a ocorrência do dano muscular os macrófagos residuais são ativados e, conjuntamente aos leucócitos infiltrados, promovem a fagocitose dos restos teciduais. Posteriormente, os macrófagos mudam seus fenótipos para resolverem a inflamação e, conjuntamente aos fatores de crescimento locais, promovem a miogênese e o crescimento da miofibrila ${ }^{(4)}$. O reparo da fibra muscular é finalizado pela transformação da célula satélite em miócitos e reunião em miotúbulos ${ }^{(12)}$. O papel do cortisol é conter (limitar) as respostas inflamatórias e imunitárias iniciais não permitindo a perpetuação do dano muscular ${ }^{(9)}$. Adicionalmente, o exercício resistido resulta em aumento nas concentrações de Gh e testosterona que estimulam maximamente as vias de síntese das proteínas miofibrilares ${ }^{(12)}$.

\section{DESADAPTAÇÕES FISIOLÓGICAS AOTREINAMENTO CONTÍNUO}

O processo de treinamento intensificado levando à supercompensação e estresse relacionado, quando visto como contínuo, sugere que 
o estresse aumentado, ou supracarga, pode resultar no rompimento da homeostase e redução temporária da função muscular.

A figura 1 enfatiza o fato que o programa de treinamento com sucesso precisa envolver não somente ampla sobrecarga (região entre as linhas pontilhadas), mas também precisa evitar a desastrosa combinação da sobrecarga excessiva com a recuperação, que resulta na instalação do estado de supratreinamento (overtraining-OT). O supratreinamento excede o supra-alcance (overreaching-OR) e resulta em desadaptação fisiológica e redução crônica no rendimento físico ${ }^{(9)}$.

O supra-alcance pode ser visto como tentativa deliberada de induzir o desempenho máximo ou como desfecho não planejado e indesejável de treinamento extenuante. Há OR quando o platô, ou decréscimo do desempenho, é revertido em poucos dias ou semanas, e é seguido de desempenho superior ou precedido pelo treinamento(9).

Os desfechos do treinamento destacam dois fatos adicionais na figura 1: i) que níveis de treinamento podem resultar em desfechos similares dependendo da natureza do treinamento e da mistura do estresse do exercício com a recuperação e regeneração e ii) atletas no pico de OR estão também no limiar do OT (ver linha pontilhada à direita). De fato, o limite entre o desempenho ótimo e o desempenho comprometido (pelo OT) é sutil ${ }^{(9)}$.

O supratreinamento procede do desbalanceamento entre treinamento e recuperação, exercício e capacidade de exercício, estresse e tolerância ao estresse, com o treinamento excedendo recuperação, o exercício excedendo a capacidade do atleta e os estressores ultrapassando a tolerância ao estresse.

No modelo de OT contínuo (Figura 1), uma vez atingido o estado de OR, um ou dois desfechos podem ocorrer. Primeiro, o atleta/técnico/ pesquisador reconhece sintomas associados ao OR e promove repouso e recuperação apropriados ao atleta. A partir disso, a completa recuperação ocorre e o processo de OR estimula o efeito supercompensatório com o desempenho se elevando a um nível maior do que o precedente. O segundo desfecho possível, seguido ao OR, é o desenvolvimento progressivo do estado de OT quando a redução do desempenho, consequente ao OT, seria motivo para aumentar o treinamento visando suprir o menor desempenho. Alternativamente, o desempenho reduzido poderia não ser detectado. Se a elevada carga de treinamento persistir e/ou o repouso e recuperação forem inadequados, haverá o estado mais grave de OT a síndrome do supratreinamento (overtraining syndrome - OTS). Outros estressores adicionais seriam competições frequentes, planos intensos de viagens, monotonia de treinamentos, estressores psicossociais e doenças/infecção(2)

Hans Selye propôs que a resposta do estresse corporal ao trabalho muscular forçado seria trifásica (figura 1). Neste contexto de Selye, a adaptação geral clássica tem como primeiro estágio o alarme, quando o corpo reconhece e reage ao estresse OR. Na fase de reação o corpo é capaz de promover adaptações fisiológicas apropriadas, sem doença ou dano de órgãos. Quando o corpo permanece sob estresse, por extenso período de tempo, o estágio de exaustão é atingido. Neste ponto, os sistemas de defesa do corpo são superados, ocorre desadaptação e a função fisiológica normal é perdida por período indefinido de tempo. As respostas hormonais estão envolvidas no três estágios em tentativa de restabelecer o equilíbrio metabólico ${ }^{(9)}$.

Assim, de acordo com Selye, a habilidade do corpo em resistir ao estresse é finita. A exposição continuada a estressores, resulta inicialmente no declínio da resistência geral (estágio da reação de alarme). Com a instalação da adaptação a resistência do corpo ao estressor se eleva acima do normal (estágio de resistência), mas com a exposição continuada ao estressor, a resistência cai a níveis subnormais permitindo o estágio de exaustão(2).

\section{MARCADORES METABÓLICOS DAS DESADAPTA- ÇÕES FISIOLÓGICAS AO TREINAMENTO CONTÍNUO}

Em consequência ao treinamento contínuo tanto OR como OT podem ser acompanhados de redução ou estagnação do desempenho. Dentre os marcadores fisiológicos mais utilizados para o acompanhamento do desempenho figura o limiar de lactato $4 \mathrm{mmol} / \mathrm{L}^{(13)}$.

Um dos marcadores clínicos do dano muscular é a dor tardia localizada (delayed onset of muscle soreness-DOMS) determinada pela palpação da barriga (belly) e região distal dos músculos vastomedial, vastolateral e retofemoral, relaxados, na posição sentado. A dor relatada é estratificada em escala de 1 (normal) a 10 (extremamente dolorido)(14). A variação da amplitude da articulação do joelho é medida como índice de edema muscular ${ }^{(15)}$. O dano morfológico muscular libera componentes intracelulares ao plasma, dentre os quais proteína (mioglobina) e enzimas (CK, LDH, AST) frequentemente utilizados como marcadores de lesão(16).

Durante o exercício extenuante, com dano muscular, inflamação e estresse oxidativo ocorrem conjuntamente. Os radicais livres são importantes tanto para a iniciação e propagação da resposta inflamatória muscular, assim como para a eclosão respiratória dos fagócitos removedores dos restos celulares do músculo danificado(11).

As reduções de glutamina ( $G \mid n$ ) e da relação glutamina/glutamato (Gln/Glu) também são consideradas bons marcadores do estresse do exercício ${ }^{(17)}$. A GIn plasmática responde ao exercício aumentando seus níveis durante o exercício intenso de curta duração (sprints ou piques) reduzindo no período de recuperação pós-exercício. A restauração dos valores pré-exercício requer várias horas, dependendo da intensidade e duração do esforço físico(18).

Treinamento extenuante e competições longas (ex. maratonas) resultam em redução da glutaminemia e requerem recuperação prolongada para restabelecimento da normalidade. Nestas circunstâncias, sistemas funcionais dependentes da glutamina como digestório e o imunitário podem ser afetados ${ }^{(17)}$.

O desarranjo da homeostase da Gln como o visto no exercício extenuante pode ser observado também em estados catabólicos como infecção, trauma e acidose metabólica ${ }^{(17)}$. Estas situações são usualmente acompanhadas do aumento no glutamato plasmático, indicador de resistência insulínica e rabdomiólise, como o observado no DM-2, câncer, $\mathrm{HIV}^{+}$e esclerose amiotrópica lateral. Nestas situações, a menor captação muscular de glutamato, conjuntamente com a elevação dos seus valores no plasma, constitui marcador do estado de pré-caquexia ${ }^{(19)}$

O treinamento excessivo acompanhado de variações no humor/ comportamento é visto como o terceiro estágio da Síndrome de Adaptação Geral de Selye como o foco orgânico sendo mais na recuperação/ sobrevivência do que na adaptação (figura 1). Os sintomas vão da excitação e inquietude (chamada forma simpática) ao comportamento fleugmático e inibitório (chamada forma parassimpática). Nos esportes de resistência (endurance) predomina a forma parassimpática ${ }^{(3)}$. Tem sido demonstrado que os distúrbios de comportamento aumentam de maneira progressiva ao aumento da carga de treinamento(20).

O método mais utilizado na exploração da relação do estado de humor com o desempenho físico é o perfil do estado de humor (Profile of Mood State-POMS). O questionário do POMS consiste de 65 adjetivos designados para a avaliação de seis estados emocionais, sentidos na semana anterior ao questionário. Os escores são então obtidos para os estados de tensão, depressão, raiva, vigor, fadiga e confusão ${ }^{(21)}$. Atletas de elite apresentam escores de POMS menores do que amostras normativas de não atletas, isto é, abaixo do percentil 50 (mediana) para os estados de depressão, tensão, raiva, fadiga e confusão, caracterizando o padrão denominado de iceberg. Por outro lado, os atletas apresentam escores acima da mediana (P50) para o estado de vigor ${ }^{(20)}$. 
Os estresses físicos ou psicológicos ativam a corticotrofina hipotalâmica, liberando a adrenocorticotrofina $(\mathrm{ACTH})$ na hipófise anterior e, consequentemente, os glicocorticoides adrenais ${ }^{(22)}$. Diferentemente da testosterona ( $\mathrm{T}$ ), o cortisol (C) é um hormônio esteroide que apresenta efeito proteolítico muscular ${ }^{(12)}$. Reduções maiores do que 30\% na relação T/C plasmática refletem o predomínio do estado catabólico sobre o anabólico muscular, constituindo índice para avaliação do treinamento e preditor do desempenho ${ }^{(23)}$.

O efeito do exercício sobre o risco e gravidade da doença infecciosa do trato aéreo superior, apresenta curva em forma de ${ }^{(24)}$. A superestimulação simpáticoadrenal e aumento do cortisol, induzida pelo treinamento exaustivo, combinadamente a restrição hídrica e ambiente quente, levam a redução do fluxo salivar e da imunoglobulina A (IgA). Consequentemente, o risco de infecções ororrespiratórias é elevado nos atletas de resistência aeróbia (25).

A elevação do cortisol tem mostrado inibir o transporte transepitelial da lgA salivar e inibir, in vivo, a síntese de anticorpos pelos linfócitos B, após o exercício. Em consequência a esses efeitos imunossupressores os atletas de resistência aeróbia (endurance) apresentam riscos aumentados de adquirirem infecções oportunistas (26).

Outra consequência importante da superestimulação simpática é sobre a redistribuição do volume plasmático e os distúrbios gastrointestinais decorrentes ${ }^{(27)}$. Um quarto a metade dos atletas de elite são acometidos de sintomas gastrointestinais induzidos pelo exercício físico. Os sintomas ocorrem em função da motilidade alterada, fatores mecânicos ou alterações das secreções neuroimunoendócrinas. A isquemia intestinal é a principal causa de náuseas, vômitos, dor abdominal e diarreia sanguinolenta. Essa isquemia pode ser precipitada quando o exercício vigoroso (com desvio da volemia visceral para músculos e pele) é praticado em ambiente quente, piorado por fatores como excitação pela competição, elevação das catecolaminas e, também, redução do esvaziamento gástrico, que pode ser agravado pelo consumo de bebidas hipertônicas durante ou após competição(27).

\section{DIAGNÓSTICO DIFERENCIAL}

Como o estabelecido por autores ${ }^{(2)}$ a diferença entre OT e OR ocorre mais pelo tempo necessário ao restabelecimento do desempenho (tabela 1) do que tipo e duração do estresse ou grau de alteração. Além disso, as definições também implicam na ausência de sinais psicológicos associados a ambas as situações. Portanto, a maior dificuldade reside na detecção da diferença sutil que pode existir nos atletas que atingiram o grau extremo da superação, gerando a presença da OTS pode existir ${ }^{(1)}$.

Tabela 1. Estágios do supra-alcance funcional (FOR) e não funcional (NFOR) e da síndrome do supratreinamento (OTS) no treinamento contínuo (adaptado de Meeusen et al., 2006(1)).

\begin{tabular}{c|c|c|c|c}
\hline Processo & Sobrecarga & Treinamento & \multicolumn{2}{|c}{ Contínuo } \\
\hline Desfecho & fadiga aguda & FOR & NFOR & OTS \\
\hline Recuperação & dia(s) & dias-semanas & semanas-meses & meses \\
\hline Desempenho & aumento & $\begin{array}{c}\text { declínio tempo- } \\
\text { rário }\end{array}$ & $\begin{array}{c}\text { estagnação, } \\
\text { diminuição }\end{array}$ & diminuição \\
\hline
\end{tabular}

Os sinônimos OTS incluem supraesforço, estagnação, fadiga crônica, suprafadiga, antiquado, queimado e OT. Durante o século 20 foram dadas varias denominações à desadaptação crônica e declínio de rendimento do estado do estado de OT incluindo síndrome do subdesempenho, síndrome da fadiga esportiva, mas, presentemente utiliza-se o termo OTS ${ }^{(9)}$.

OTS é a forma crônica de desadaptação fisiológica ao treinamento. A OTS é um conjunto de sintomas físicos e psicológicos, persistentes, que ocorrem, subsequentemente, à aplicação de cargas pesadas de treinamento. Dentre os mais de 90 sinais e sintomas listados para a OTS estão o decréscimo do desempenho físico, fadiga geral, indisposição, insônia, anorexia, irritabilidade, excitabilidade, ansiedade, perda de vigor, inquie- tude, perda de peso, perda de motivação, perda da concentração mental e sensação de depressão. Entretanto, os fatores críticos ao diagnóstico de OTS incluem a redução crônica do desempenho prolongada por meses ou anos ${ }^{(1)}$ e não simplesmente a existência de sinais e sintomas. Alguns atletas com OTS exibem apenas poucos sinais enquanto outros nem mesmo apresentam algum ${ }^{(9)}$.

O simples reconhecimento da OTS é complicado por i)variação interindivíduos considerável, em relação ao desenvolvimento de OT; ii) os sinais e sintomas de OTS diferem na redução dos desempenhos agudo vs crônico; iii) o volume excessivo de treinamento pode afetar o corpo de forma diferente da intensidade excessiva de treinamento e iiii) a OTS apresenta dois tipos de respostas autossômicas. A forma simpática exibe insônia, irritabilidade, inquietação e taquicardia enquanto a forma parassimpática apresenta fadiga, depressão, apatia e bradicardia. Ambas formas de OTS envolvem queda do desempenho. O tipo simpático é raro e afeta, supostamente, atletas de esportes anaeróbios que envolvam piquem, saltos e arremessos. O tipo parassimpático afeta atletas de resistência altamente treinados em esportes aeróbios tais como corrida de longa distância, natação e ciclismo ${ }^{(9)}$.

Funcionários estressados, alunos em exames e cavalos de corrida exibem sinais e sintomas similares ao observados em atletas com OTS, o que é consistente com o conceito que a OTS reflete a tentativa do corpo humano em lutar com os estresses psicológico e não-psicológico. A OTS representa a soma de múltiplos estressores da vida tais como treinamento físico, perda de sono, exposição a estresses ambientais etc.

\section{CONCEPÇÕES ERRÔNEAS DE TERMINOLOGIA E DE DEFINIÇÃO}

De acordo com o treinamento contínuo, o atleta que se submete ao treinamento intensificado, na ausência de recuperação apropriada, pode não responder positivamente ao treinamento (figura 1), dando lugar à fadiga progressiva e redução do desempenho, como resultantes a uma simples sessão de treinamento intenso, identificados como OR ou então OT ${ }^{(2)}$.

Supra-alcance (OR) é definido como acúmulo de estresse de treinamento e/ou não treinamento resultando em decréscimo a curto prazo na capacidade de desempenho com ou sem sinais fisiológicos e psicológicos relacionados. A restauração da capacidade de desempenho pode levar vários dias a semanas. Entretanto, se o estresse do treinamento e/ ou não treinamento for contínuo, poderá resultar no estado de OT.

O supratreinamento é definido como acumulo do estresse do treinamento e/ou não treinamento resultando no longo decréscimo da capacidade de desempenho com ou sem sinais fisiológicos e psicológicos relacionados, em que a restauração da capacidade de desempenho pode levar varias semanas ou meses ${ }^{(2)}$

Baseados nestas definições ORe OT são relacionadas não apenas a redução da capacidade de desempenho, mas também aos sinais fisiológicos e psicológicos. A diferença entre esses dois fenômenos está na quantidade de tempo necessária para restauração do desempenho e no desfecho clinico e não, ao tipo de duração do exercício e grau de alteração(2).

As seguintes características podem ocorrer nas formas de OT: acúmulo de fadiga, decorrente ou não do exercício, estagnação ou redução do desempenho no limiar do lactato $(4 \mathrm{mmol} / \mathrm{L})$, incompetência competitiva, distúrbios do estado de humor, dor/inchaço muscular na capacidade de desempenho máximo.

A excreção urinária de norepinefrina reduzida em mais de 60\% é indicativa de OT enquanto variações menores que $40 \%$ caracterizam $O R^{(28)}$. Nenhum dos vários marcadores bioquímicos do OT pode ser universalizado. 0 único critério confiável para diagnóstico de OT éa perda do nível de desempenho apesar da manutenção ou aumento da carga de treinamento ${ }^{(3)}$.

Tem sido anedoticamente sugerido que o OR pode ocorrer mais em esportes de equipes e em esportes de potência/torque, enquanto que 
OT seria mais prevalente em esportes de resistência. Semelhantemente, o grau de insuficiência experimentado no OT tem expectativa de ser significativamente pior comparativamente ao estado de $\mathrm{OR}^{(2)}$.

Embora a fundamentação do OT contínuo pareça lógica, não há evidências confirmando que OR evolua para OT ou que sintomas de OT sejam piores do que os de $O R^{(2)}$.

Tanto as definições de OR e OT não são inteiramente satisfatórias(2). O Colégio Europeu de Ciências dos Esportes possui definições mais detalhadas $^{(1)}$. Considera OT como processo contínuo de treinamento intensificado com possíveis desfechos a curto e longo prazo. O desfecho de supra-alcance (OR) a curto prazo é denominado OR funcional (FOR) e a longo prazo ou extremo OR é conhecido por OR não funcional (NFOR). A forma crônica de desadaptação metabólica é chamada OTS ${ }^{(1)}$. As três formas têm em comum a redução da capacidade de desempenho ea relativa incompetência competitiva ${ }^{(3)}$. Diferem, entretanto, no tempo de recuperação e prognóstico (tabela 1). A FOR necessita de período relativamente curto para recuperação (dias até duas semanas), apresenta boa possibilidade de efeitos supercompensatórios e prognóstico favorável ${ }^{(2,3)}$.

ANFOR refere-se ao declínio do desempenho que pode ser revertido por período regenerativo de semanas ou meses. Por sua vez, o declínio

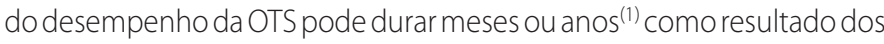
estresses, do treinamento com exercícios prolongados, aplicados cronicamente e concorrentemente com recuperação inadequada ${ }^{(9)}$. Ambos NFOR e OTS apresentam prognóstico desfavorável|(3). Entretanto, exceto informações anedóticas, não há evidências correntes que sugiram ser FOR precedente a NFOR e de que os sintomas de NFOR sejam mais graves do que os do FOR. No entanto, as evidencias disponíveis, tanto anedóticas como científicas confirmam a existência de OTS ${ }^{(3)}$.

Para evitar conceitos terminológicos errôneos, OT é um processo de treinamento intensificado com possíveis desfechos de OR a curto prazo (FOR), extremo OR (NFOR) ou OTS. A expressão síndrome (OTS) enfatiza a etiologia multifatorial do estado e reconhece que o exercício (treinamento) não é, necessariamente, o único fator causativo da síndrome ${ }^{(1)}$.

A OTS reflete a tentativa de luta do corpo humano com estressores fisiológicos ou não, adicionais ao treinamento físico, envolvendo perda de sono, estresses ambientais (calor, umidade, frio, alta altitude) pressões ocupacionais e mudanças de residência. Desta forma, a OTS pode ser entendida, parcial-

\section{REFERÊNCIAS}

1. Meeusen R, Duclos M, Gleeson M, Rietjens G, Steinacker J, Urhausen A. Prevention, diagnosis and treatment of the Overtraining Syndrome European. J Sports Sci 2006;6:1-14.

2. Halson SL, Jeukendrup AE. Does overtraining exist? An analysis of overreaching and overtraining research. Sports Med 2004;34:967-81.

3. Lehmann M, Foster C, Keul J. Overtraining in endurance athletes: a brief review. Med Sci Sports Exerc 1993;25:854-62.

4. Chalab H, Chernaoul M, Gomez-Merino D, Drogon C, Santivel S, Guezennec C. Étude des manifestations neuroencodriniennes et ortho-parasympathiques de la fatigue dans le cadre du snivi biologique de l'athlete de hant nivean. Exemple chez les foot balleurs professionnels du Paris Saint-Germain. Revue Francophone des Laboratoires 2005:375:21-7.

5. Reilly $\mathrm{T}$. Energetics of high-intensity exercise (soccer) with particular reference to fatigue. J Sports Sci 1997; 15:257-63.

6. Fry RW, Morton AR, Keast D. Overtraining in athletes. An update. Sports Med 1991;12:32-65.

7. Christensen NJ, Galbo H. Sympathetic nervous activity during exercise. Annu Rev Physiol 1983;45:139-53

8. Roatta S, Farina D. Sympathetic actions on the skeletal muscle. Exerc Sport Sci Rev 2010;38:31-5.

9. Armstrong $L E, V a n H e e s t ~ J L$. The unknown mechanism of the overtraining syndrome: clues from depression and psychoneuroimmunology. Sports Med 2002;32:185-209

10. Robergs RA, Roberts SO. Função neuromuscular e adaptaçăo ao exercício. In: Princípios fundamentais de fisiologia do exercício para adaptação, desempenho e saúde. 1a ed. Brasileira, São Paulo: Phorte Editora; 2002: p. 76-109.

11. Tiidus PM. Radical species in inflammation and overtraining. Can J Physiol Pharmacol 1998;76:533-8.

12. Manda RM, Maestá N, Burini RC. Bases metabólicas do crescimento muscular. Rev Bras Fisiol Exerc 2010;9:16-27.

13. Heck H, Mader A, Hess G, Mucke S, Muller R, Hollmann W. Justification of the 4-mmol// lactate threshold. Int J Sports Med 1985;6:117-30.

14. Fatouros IG, Destouni A, Margonis K, Jamurtas AZ, Vrettou C, Kouretas D, et al. Cell-free plasma DNA as a nove marker of aseptic inflammation severity related to exercise overtraining. Clin Chem 2006;52:1820-4.

15. Clarkson PM, Tremblay I. Exercise-induced muscle damage, repair, and adaptation in humans. J Appl Physiol 1988;65:1-6.

16. Petricio AIM, Porto M, Burini RC. Alterações hemodinâmicas do equilíbrio ácido básico e enzimáticas no exercício exaustivo com pesos. Rev Bras Ativ Fis Saúde 2001;6:17-26. mente, dentro do contexto da Síndrome de Adaptação Geral de Selye ${ }^{(2)}$.

A etiologia da OTS envolve a exclusão de doenças orgânicas ou infecções e fatores dietéticos como restrição calórica, deficiências de carboidratos, e/ou proteína, ferro e magnésio, que funcionariam como gatilho. Um dos gatilhos mais certos da OTS é o erro técnico, resultando em desbalanço entre carga de treinamento e recuperação. Outros possíveis gatilhos seriam a monotonia do treinamento, excesso de competições, problemas pessoais e emocionais (psicológico) e demandas emocionais do desafio. Adicionalmente, exposição à altitude, estresse térmico, depleção de glicogênio muscular e infecções funcionariam como gatilhos da OTS ${ }^{(1)}$.

NFOR e OTS são muito difíceis de serem diferenciados em humanos e podem estar associados a um ou mais dos seguintes sintomas: estado catabólico acentuado com possível perda de massa corporal(3), imunossupressão e elevada incidência de agressão e alterações de humor ${ }^{(1,2)}$.

Além do diagnóstico baseado na recuperação do desempenho (mais curto na NFOR do que na OTS) ${ }^{(1,2)}$ outras distinções dependem do desfecho clínico e diagnóstico de exclusão de infecções orgânicas ou doenças nutricionais, mais comuns na OTS(4).

Não há biomarcador objetivo para OTS. Sua confirmação requer variações nos valores hormonais ou da bioquímica sanguínea ${ }^{(3)}$. O período de intenso treinamento pode intensificar e/ou prolongar a resposta inflamatória musculoarticular com elevadas consequências catabólicas ou imunossupressão(29).

Assim, considerando-se que não há instrumento diagnóstico para identificar se o atleta está sofrendo de OTS, o diagnóstico definitivo de OTS requer sempre a exclusão de outras doenças orgânicas incluindo endócrinas (tireoide, suprarenal e diabetes), deficiência de ferro e anemia ou doenças infecciosas, incluindo miocardite, hepatite e parotidite. Também os distúrbios alimentares como anorexia nervosa e bulimia precisam ser excluídos ${ }^{(1)}$. Presentemente, é impossível a diferenciação da fadiga aguda ou queda de desempenho, experimentados em sessões isoladas de treinamento, dos estados de OR e OT. Isto é, parcialmente, resultado da falta de instrumentos diagnóstico (propedêutica), variabilidade dos resultados dos estudos, das respostas individuais ao treinamento e falta de estudos bem controlados.

Todos os autores declararam não haver qualquer potencial conflito de interesses referente a este artigo.

17. Rowbottom DG, Keast D, Morton AR. The emerging role of glutamine as an indicator of exercise stress and overtraining. Sports Med 1996;21:80-97

18. Ispirlidis I, Fatouros IG, Jamurtas AZ, Nikolaidis MG, Michailidis I, Douroudos I, et al. Time-course of changes in inflammatory and performance responses following a soccer game. Clin J Sport Med 2008;18:423-31.

19. Hack V, Stutz O, Kinscherf R, Schykowski M, Kellerer M, Holm E, et al. Elevated venous glutamate levels in (pre)catabolic conditions result at least partly from a decreased glutamate transport activity. J Mol Med 1996;74:337-43.

20. Filaire E, Bernain X, Sagnol M, Lac G. Preliminary results on mood state, salivary testosterone:cortisol ratio and team performance in a professional soccer team. Eur J Appl Physiol 2001;86:179-84.

21. McNair D, Lorr M, Droppleman L. Profile of mood stages manual. Educational and industrial testing service, San Diego, Calif., 1971

22. Moreira A, Arsati F, de Oliveira Lima ArsatiYB, da Silva DA, de Araujo VC. Salivary cortisol in top-level professional soccer players. Eur J Appl Physiol 2009;106:25-30.

23. Handziski Z, Maleska V, Petrovska S, Nikolik S, Mickoska E, Dalip M, et al. The changes of ACTH, cortisol, testosterone and testosterone/cortisol ratio in professional soccer players during a competition half-season. Bratisl Lek Listy 2006;107:259-63.

24. Nieman DC, Johanssen LM, Lee JW. Infectious episodes in runners before and after a roadrace. J Sports Med Phys Fitness 1989;29:289-96.

25. Walsh NP, Montague JC, Callow N, Rowlands AV. Saliva flow rate, total protein concentration and osmolality as potential markers of whole body hydration status during progressive acute dehydration in humans. Arch Oral Biol 2004;49:149-54.

26. Gleeson M, McDonald WA, Pyne DB, Cripps AW, Francis JL, Fricker PA, et al. Salivary lgA levels and infection risk in elite swimmers. Med Sci Sports Exerc 1999;31:67-73.

27. de Oliveira EP, Burini RC. The impact of physical exercise on the gastrointestinal tract. Curr Opin Clin Nutr Metab Care 2009;12:533-8.

28. Naessens G, Chandler TJ, KiblerWB, Driessens M. Clinical usefulness of nocturnal urinary noradrenaline excretion patterns in the follow-up of training processes in high-level soccer players. J. strength \& Conditioning Res 2000;14:125-31.

29. Smith LL. Cytokine hypothesis of overtraining: a physiological adaptation to excessive stress? Med Sci Sports Exerc 2000;32:317-31. 